TRI-PP-00-03

Feb 2000

\title{
Hyperfine effects in charmed baryons
}

\author{
R.M. Woloshyn \\ TRIUMF, 4004 Wesbrook Mall, Vancouver, BC, Canada V6T 2A3
}

\begin{abstract}
Hadron masses are calculated in quenched lattice QCD with a fermion action of the D234 type on an anisotropic lattice. Hyperfine splittings for singly charmed baryons are found to be in agreement with expectations from the quark model and with a magnitude slightly larger than experimental values. Masses of doubly charmed baryons are also calculated and compared to a variety of model calculations. Hyperfine splittings in doubly charmed baryons are found to be slightly smaller than in singly charmed states.
\end{abstract}




\section{Introduction}

Relatively little work has been done on heavy baryons using lattice QCD. The most complete study in a relativistic framework is the one done in the UKQCD collaboration[1] for both charm and bottom flavoured baryons using an $O(a)$ improved fermion action in quenched approximation. In that work hyperfine splittings in both charm and bottom baryons were found to be considerably smaller than those predicted by phenomenological models [2] and observed experimentally. A recent NRQCD calculation 3 was able to resolve the hyperfine splittings for baryons with b quarks and found values in accord with phenomenological expectations. Since the hyperfine splitting is an important feature of the baryon spectrum further investigation of charmed baryons seems justified.

In this work we present the results of another quenched lattice simulation for charmed baryons. Due to limitations in computing resources it was not possible to use the same lattice spacing and volume as used in the UKQCD calculation. Rather we work on a more coarse lattice $(\sim 0.2 \mathrm{fm})$ with a highly improved action. Past experience has shown that results of reasonable accuracy may be obtained with such lattices [4. In order to check the calculation, the spectrum of baryons in the light quark $(\mathrm{u}, \mathrm{d}, \mathrm{s})$ sector was calculated at the same time. As well, meson masses for both heavy and light quarks were calculated. The results of all these calculations are in reasonable agreement with experimental values and with the results obtained at small lattice spacing[5, 6].

The simulation reported here differs from [1] in two other respects. In (11) the interpolating operators used for the baryons were taken to have a form which emphasizes the heavy quark symmetry. Secondly, the spin $1 / 2$ and spin $3 / 2 \sum$-like baryons are interpolated by the same operator, a RaritaSchwinger spin-3/2 field. As is well known\|7], the correlator of such a field propagates both $J=1 / 2$ and $J=3 / 2$ states and it is the $J=1 / 2$ projection of this correlator that is identified with the $\Sigma$-like baryons in [罒.

The procedure used here is different. As has been done in the context of a QCD sum rule calculation[8], the interpolating fields used for heavy baryons are taken to have the same form as those used in the light quark sector. The $J=1 / 2 \Sigma$ baryon has an interpolating operator which is distinct from that used for the $J=3 / 2 \Sigma^{*}$, just as the nucleon is usually interpolated by a field that is distinct from the $\Delta$. No assumption is made about heavy quark

symmetry. An advantage of this is that we can use the same procedure (and 
computer code) at all masses which provides some check on the results.

The correlation function used to extract the masses of $\Sigma$ 's is not obtained from the projection of the $J=3 / 2$ field's correlator. In fact, it seems that the

spin $1 / 2$ projection of this correlator has very poor overlap with the $J=1 / 2$ ground state. Our finding is that the spin $1 / 2$ projected correlator is very small and noisy compared to the correlator calculated directly using a spin $1 / 2$ field.

The final conclusion of this study is that in quenched lattice QCD the hyperfine splittings for both singly and doubly charmed baryons are in reasonable agreement with phenomenological expectations. Indications are that the splittings may be overestimated compared to experiment which seems to be a common tendency for quenched QCD simulations of baryons at all quark masses.

\section{Method}

The calculation is done on an anisotropic lattice [9 using the gauge field action

$$
\begin{aligned}
S_{G}(U)= & \beta\left[c_{p s} \sum_{p s}\left(1-\frac{1}{3} \operatorname{Re} \operatorname{Tr} U_{p s}\right)+c_{r s} \sum_{r s}\left(1-\frac{1}{3} \operatorname{Re} \operatorname{Tr} U_{r s}\right)\right. \\
& +c_{p t} \sum_{p t}\left(1-\frac{1}{3} \operatorname{Re} \operatorname{Tr} U_{p t}\right)+c_{r s t} \sum_{r s t}\left(1-\frac{1}{3} \operatorname{Re} \operatorname{Tr} U_{r s t}\right) \\
& \left.+c_{r t s} \sum_{r t s}\left(1-\frac{1}{3} \operatorname{Re} \operatorname{Tr} U_{r t s}\right)\right]
\end{aligned}
$$

where ps and rs denote spatial plaquettes and spatial planar $2 \times 1$ rectangles respectively. The plaquettes lying in the temporal-spatial planes are denoted by pt while rectangles with the long side in a spatial(temporal) direction are labeled by rst(rts). The c coefficients incorporate the aspect ratio $\xi=a_{s} / a_{t}$ and gauge link renormalization factors $u_{s}$ and $u_{t}$. These renormalization factors are estimated using the link expectation value in Landau gauge.

The fermion action is of the anisotropic D234 type 10 and has the form

$$
S_{F}=\sum_{x, i}\left(c_{1 i} D_{1 i}(x)+c_{2 i} D_{2 i}(x)\right)+\sum_{x}\left(c_{1 t} D_{1 t}(x)+c_{2 t} D_{2 t}(x)\right)
$$


Table 1: Coefficients appearing in the gauge and fermion actions

$$
\begin{aligned}
& \begin{array}{ccccc} 
& \mathrm{s} & \mathrm{t} & \mathrm{st} & \mathrm{ts} \\
\hline c_{p \ldots} & \frac{5}{3 u_{s}^{4} \xi} & \frac{5 \xi}{3 u_{s}^{2} u_{t}^{2}} & &
\end{array} \\
& c_{r \ldots} \quad \frac{-1}{12 u_{s}^{6} \xi} \quad \frac{-\xi}{12 u_{s}^{4} u_{t}^{2}} \quad \frac{-\xi}{12 u_{s}^{2} u_{t}^{4}} \\
& c_{0 \ldots} \quad \frac{2 \kappa}{3 u_{s}^{4} \xi^{2}} \quad \frac{2 \kappa}{3 u_{s}^{2} u_{t}^{2} \xi} \\
& c_{1 \ldots} \quad \frac{4 \kappa}{3 u_{s} \xi^{2}} \quad \frac{4 \kappa}{3 u_{t}} \\
& c_{2 \ldots} \quad \frac{-\kappa}{6 u_{s}^{2} \xi^{2}} \quad \frac{-\kappa}{6 u_{t}^{2}} \\
& +\sum_{x, i<j} c_{0 s} \bar{\psi}(x) \sigma_{i j} F_{i j}(x) \psi(x)+\sum_{x, i} c_{0 t} \bar{\psi}(x) \sigma_{0 i} F_{0 i}(x) \psi(x) \\
& -\sum_{x} \bar{\psi}(x) \psi(x)
\end{aligned}
$$

where

$$
\begin{gathered}
D_{1 i}(x)=\bar{\psi}(x)\left(1-\xi \gamma_{i}\right) U_{i}(x) \psi(x+\widehat{i})+\bar{\psi}(x+\widehat{i})\left(1+\xi \gamma_{i}\right) U_{i}^{\dagger}(x) \psi(x), \\
\begin{aligned}
D_{1 t}(x)=\bar{\psi}(x)\left(1-\gamma_{4}\right) & U_{4}(x) \psi(x+\widehat{t})+\bar{\psi}(x+\widehat{t})\left(1+\gamma_{4}\right) U_{4}^{\dagger}(x) \psi(x), \\
D_{2 i}(x)= & \bar{\psi}(x)\left(1-\xi \gamma_{i}\right) U_{i}(x) U_{i}(x+\widehat{i}) \psi(x+2 \widehat{i}) \\
& +\bar{\psi}(x+2 \hat{i})\left(1+\xi \gamma_{i}\right) U_{i}^{\dagger}(x+\widehat{i}) U_{i}^{\dagger}(x) \psi(x), \\
D_{2 t}(x)= & \bar{\psi}(x)\left(1-\gamma_{4}\right) U_{4}(x) U_{4}(x+\widehat{t}) \psi(x+2 \widehat{t}) \\
& +\bar{\psi}(x+2 \hat{t})\left(1+\gamma_{4}\right) U_{4}^{\dagger}(x+\widehat{t}) U_{4}^{\dagger}(x) \psi(x) .
\end{aligned}
\end{gathered}
$$

The c coefficients in the fermion action include the aspect ratio, link renormalization and the hopping parameter factors and are shown in Table 1 .

Hadron masses are calculated from zero-momentum correlation functions in the usual way. For mesons the interpolating fields were just the standard ones. For baryons some discussion is needed since the procedure used here differs from that used in [1]. Start from the light quark $(\mathrm{u}, \mathrm{d}, \mathrm{s})$ sector. A common choice 11 for the proton operator in terms of $u$ and $d$ quark fields is

$$
\epsilon^{a b c}\left[u_{a}^{T} C \gamma_{5} d_{b}\right] u_{c}
$$


where a,b,c are colour indices and Dirac indices have been suppressed. For $\Delta$ the operator

$$
\frac{1}{\sqrt{3}} \epsilon^{a b c}\left\{2\left[u_{a}^{T} C \gamma_{\mu} d_{b}\right] u_{c}+\left[u_{a}^{T} C \gamma_{\mu} u_{b}\right] d_{c}\right\}
$$

is used. This choice of operators is not unique[12] but it allows an easy generalization to other baryons[1]]. The interpolating operators for the strange hyperons $\Sigma$ and $\Sigma^{*}$ are obtained by the replacement $d \rightarrow s$ in (7) and (8) respectively. Similarly the interpolators for $\Xi$ and $\Xi^{*}$ are constructed by the replacement $u \rightarrow s$. The $\Lambda$ hyperon is more of a problem. In the $\mathrm{SU}(3)$ flavour limit it would be natural to use the octet lambda

$$
\Lambda_{8}=\frac{1}{\sqrt{6}} \epsilon^{a b c}\left\{2\left[u_{a}^{T} C \gamma_{5} d_{b}\right] s_{c}+\left[u_{a}^{T} C \gamma_{5} s_{b}\right] d_{c}-\left[d_{a}^{T} C \gamma_{5} s_{b}\right] u_{c}\right\}
$$

since it is degenerate with the nucleon and $\Delta$. However, since $\mathrm{SU}(3)$ flavour is broken, this choice is not compelling. For example, in [11] a combination $\Lambda$ with both $\mathrm{SU}(3)$ octet and singlet components was defined. In this work a "heavy" $\Lambda$ with a form

$$
\left[u_{a}^{T} C \gamma_{5} d_{b}\right] s_{c},
$$

which is natural in the heavy quark limit, is also used.

The operators used to calculate the masses of the eight ground state singly-charmed baryons are taken to have the same structure as the operators given above. The $\Sigma_{c}$ and $\Sigma_{c}^{*}$ baryons are obtained by the substitution $d \rightarrow c$ in (7) and (8) while $\Omega_{c}$ and $\Omega_{c}^{*}$ are obtained by $u \rightarrow s, \mathrm{~d} \rightarrow c$. For the $\Lambda_{c}$ and $\Xi_{c}$ the operators (9) and (10) with the replacements $s \rightarrow c$ and $d \rightarrow s, s \rightarrow c$ are used. Finally, for the remaining two states we take

$$
\Xi_{c}^{\prime}=\frac{1}{\sqrt{2}} \epsilon^{a b c}\left\{\left[u_{a}^{T} C \gamma_{5} c_{b}\right] s_{c}+\left[s_{a}^{T} C \gamma_{5} c_{b}\right] u_{c}\right\}
$$

and

$$
\Xi_{c}^{*}=\frac{2}{\sqrt{3}} \epsilon^{a b c}\left\{\left[u_{a}^{T} C \gamma_{\mu} s_{b}\right] c_{c}+\left[s_{a}^{T} C \gamma_{\mu} c_{b}\right] u_{c}+\left[c_{a}^{T} C \gamma_{\mu} u_{b}\right] s_{c}\right\} .
$$

Rather than using the above operators which have an explicit relativistic form one could consider the operators which survive in the limit of a static charm quark. The way to do this has been discussed in some detail in the context of QCD sum rule calculations 13 , 14. However there is no particular advantage to taking this limit here. The operators we use contain the leading 
heavy-quark components so the simulation will decide by itself whether they are dominant. The advantage of using the explicit relativistic forms is that it allows a unified analysis of hyperfine effects over the whole mass range from nucleon and $\Delta$ to charmed baryons.

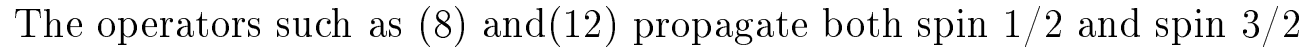
states [7]. At zero momentum the correlation function with spatial Lorentz indices has the general form

$$
C_{i j}(t)=\left(\delta_{i j}-\frac{1}{3} \gamma_{i} \gamma_{j}\right) C_{3 / 2}(t)+\frac{1}{3} \gamma_{i} \gamma_{j} C_{1 / 2}(t)
$$

where the subscripts $3 / 2$ and $1 / 2$ denoted the spin projections. The quantity $C_{3 / 2}(t)$ was used to extract the mass of the spin $3 / 2$ states. However, it was found that the spin $1 / 2$ projection $C_{1 / 2}(t)$ was too noisy at large time separations to allow for the determination of a mass.

Hadron correlators were calculated using interpolating operators in local form at both source and sink and also applying a gauge invariant smearing to the quark propagators at the sink. The Gaussian smearing function, eqn(13)

of [15], was used. Hadron masses were obtained by a simultaneous fit to local and sink-smeared correlators.

\section{Results}

The calculations were carried out at $\beta=2.1$ on lattices with a bare aspect ratio $\xi$ of 2 . The Landau link tadpole factors were first determined iteratively to be $u_{s}=0.7858$ and $u_{t}=0.9472$ and these values were used in all subsequent calculations. The static potential was determined from both spatial and spatial-temporal Wilson loops. From this the lattice spacing and renormalized anisotropy were obtained. The results for the lattice spacings are $a_{s}^{-1}=(0.977 \pm 0.003) G e V$ and $a_{t}^{-1}=(1.914 \pm 0.017) G e V$ with a systematic uncertainty of $0.01 \mathrm{GeV}$ coming from uncertainty in the choice of parametrization of the short distance part of the potential[|[6]. The renormalized anisotropy was found to be $1.95 \pm 0.02$ which is compatible with other studies done with improved gluon actions at similar lattice spacings 17 .

Fermion propagators were calculated on a $10^{3} \times 30$ lattice with Dirichlet boundary conditions on the fermion fields. A total of 420 configurations were analyzed. With some preliminary tuning it was found that $\kappa=0.182$ and $\kappa=0.237$ gave good values for the $J / \psi$ and $\phi$ meson masses so these were the 
Table 2: Hadron masses for light quarks. Masses are given in $\mathrm{GeV}$, mass differences are in $\mathrm{MeV}$. The experimental values in this and other tables are from 18

\begin{tabular}{ccccc}
\hline & This work & CP-PACS[5] & UKQCD[6] & Experiment \\
\hline$K$ & $0.485(6)$ & $0.553(10)$ & & 0.498 \\
$K^{*}$ & $0.902(26)$ & $0.889(3)$ & $0.748_{-46}^{+81}$ & 0.896 \\
$N$ & $0.942(67)$ & $0.878(25)$ & & 0.940 \\
$\Delta$ & $1.358(71)$ & $1.257(35)$ & $1.25_{-9}^{+19}$ & 1.232 \\
$\Lambda$ & $1.105(46)$ & $1.060(13)$ & $1.088_{-19}^{+20}$ & 1.116 \\
$\Sigma$ & $1.184(35)$ & $1.176(11)$ & $1.091_{-11}^{+22}$ & 1.193 \\
$\Sigma^{*}$ & $1.488(57)$ & $1.388(24)$ & $1.38_{-7}^{+15}$ & 1.384 \\
$\Xi$ & $1.283(28)$ & $1.288(8)$ & $1.242_{-24}^{+43}$ & 1.318 \\
$\Xi^{*}$ & $1.590(39)$ & $1.517(16)$ & $1.51_{-5}^{+11}$ & 1.534 \\
$K^{*}-K$ & $417(27)$ & & & 398 \\
$\Delta-N$ & $416(93)$ & & & 292 \\
$\Sigma^{*}-\Sigma$ & $304(64)$ & & & 191 \\
$\Xi^{*}-\Xi$ & $307(43)$ & & & 216 \\
\hline
\end{tabular}

$\kappa$ values adopted for the charm and strange quarks for all calculations. Where necessary, masses were extrapolated to the physical up and down quark region using results from the set of hopping parameter values $0.229,0.233,0.237$ and 0.241 . The value of the critical $\kappa$ is $0.2429(2)$.

First consider the light quark $(\mathrm{u}, \mathrm{d}, \mathrm{s})$ sector. The pion and $\rho$-meson masses were fixed at $0.140 \mathrm{GeV}$ and $0.770 \mathrm{GeV}$ which determines the hopping parameter for up and down quarks (taken to be degenerate) and the lattice scale $a_{\rho}$. It was found that $a_{\rho}^{-1}=(1.99 \pm 0.12) G e V$ which is slightly larger than $a_{t}^{-1}$ found from the static potential. This is an inevitable result of the quenched approximation. The $\rho$-meson mass scale was used in all subsequent calculations. The results in the light quark sector are given in Table 2 with statistical errors obtained by a bootstrap procedure. The dominant systematic error, a $6 \%$ uncertainty in the scale determination, is not shown explicitly in this and subsequent tables but should be kept in mind. For comparison, results from recent calculations (Table II in [5] and Table XVII in |6]) done at small lattice spacing and extrapolated to the continuum are also shown. The results of our coarse lattice simulation are seen to be quite reasonable.

In Table 2 the $\Lambda$ mass calculated with the operator (9) is given. The mass obtained using (10) was essentially identical. This was found to be true for 
Table 3: Masses for mesons with charm quarks. Masses are given in $\mathrm{GeV}$, mass differences are in $\mathrm{MeV}$.

\begin{tabular}{ccc}
\hline & This work & Experiment \\
\hline$\eta_{c}$ & $3.012(4)$ & 2.980 \\
$J / \psi$ & $3.087(4)$ & 3.097 \\
$D$ & $1.875(6)$ & 1.867 \\
$D^{*}$ & $2.007(9)$ & 2.008 \\
$D_{s}$ & $1.965(4)$ & 1.969 \\
$D_{s}^{*}$ & $2.086(6)$ & 2.112 \\
$J / \psi-\eta_{c}$ & $78(2)$ & 117 \\
$D^{*}-D$ & $136(7)$ & 141 \\
$D_{s}^{*}-D_{s}$ & $122(4)$ & 143 \\
\hline
\end{tabular}

all quark masses.

As check on how well charmed quarks are being simulated we first show the results for charmonium and D-mesons in Table 3 . The hyperfine splitting between $\eta_{c}$ and $J / \psi$ is 78(2)(5) MeV which is in very good agreement with the results obtained in [19] using a completely different fermion action. It is considerably smaller than the experimental value which is a well known feature of quenched QCD simulations for quarkonium[20]. The $D^{*}-D$ splittings are compatible with results from NRQCD on similar lattices 21 and are also smaller than experimental values although the suppression of hyperfine effects is less pronounced than in charmonium.

As mentioned in Section 2 the interpolating operators used for charmed baryons are taken to have the same form as those used for the light baryons. For example, the correlator for $\Sigma_{c}$ is calculated directly using operators which are the same as used for the strange $\Sigma$ hyperon except the mass is increased to charm. An alternative is to extract the masses of $\Sigma$-like baryons (e.g., $\left.\Sigma_{c}, \Omega_{c}\right)$ from the spin-1/2 projection of a correlation function of spin $3 / 2$ fields( see (13)). Figure 1 illustrates why this alternative is not used here. The correlation functions for $\Omega_{c}$ and the spin-3/2 projected $\Omega_{c}^{*}$ are plotted as a function of lattice time. Also shown is the spin- $1 / 2$ projected correlator. This spin- $1 / 2$ projected piece has a very fast pre-asymptotic falloff and is therefore small and noisy in the time region in which one would want to extract the mass. This shows that the overlap of the spin- $1 / 2$ projection is small. It is also worth noting from Fig. 1 that even without any analysis one sees that the $\Omega_{c}$ correlator has a less rapid falloff than that of $\Omega_{c}^{*}$ i.e., $\Omega_{c}$ is 
Table 4: Masses of singly charmed baryons. Masses are given in $\mathrm{GeV}$, mass differences are in $\mathrm{MeV}$. The experimental values are taken from 18 except for $\Xi_{c}^{\prime}$ which is from [22]

\begin{tabular}{cccc}
\hline & This work & UKQCD[1] & Experiment \\
\hline$\Lambda_{c}$ & $2.304(39)$ & $2.27_{-3}^{+4}+3$ & $2.285(1)$ \\
$\Sigma_{c}$ & $2.465(22)$ & $2.46_{-3}^{+7}+5$ & $2.453(1)$ \\
$\Sigma_{c}^{*}$ & $2.557(30)$ & $2.44_{-4}^{+6}{ }_{-5}^{+4}$ & $2.518(2)$ \\
$\Xi_{c}$ & $2.454(21)$ & $2.41_{-3}^{+3}{ }_{-4}^{+4}$ & $2.468(2)$ \\
$\Xi_{c}^{\prime}$ & $2.579(14)$ & $2.51_{-3}^{+6}{ }_{-6}^{+6}$ & $2.575(3)$ \\
$\Xi_{c}^{*}$ & $2.672(16)$ & $2.55_{-4}^{+5}+6$ & $2.645(2)$ \\
$\Omega_{c}$ & $2.664(12)$ & $2.68_{-4}^{+5}{ }_{-6}^{+5}$ & $2.704(4)$ \\
$\Omega_{c}^{*}$ & $2.757(14)$ & $2.66_{-3}^{+5}{ }_{-7}^{+6}$ & \\
$\Sigma_{c}^{*}-\Sigma_{c}$ & $91(25)$ & $-17_{-31}^{+12}{ }_{-2}^{+3}$ & $65(2)$ \\
$\Xi_{c}^{*}-\Xi_{c}^{\prime}$ & $94(13)$ & $-20_{-24}^{+12}+2$ & $70(4)$ \\
$\Omega_{c}^{*}-\Omega_{c}$ & $94(10)$ & $-23_{-14}^{+6}{ }_{-2}^{+3}$ & \\
\hline
\end{tabular}

Table 5: Masses of doubly charmed baryons in $\mathrm{GeV}$

\begin{tabular}{ccccc}
\hline & $\Xi_{c c}$ & $\Xi_{c c}^{*}$ & $\Omega_{c c}$ & $\Omega_{c c}^{*}$ \\
\hline This work & $3.598(13)$ & $3.682(20)$ & $3.697(10)$ & $3.775(12)$ \\
Potential model[23] & 3.478 & 3.61 & & \\
Mass formulae[24] & $3.610(7)$ & $3.735(17)$ & $3.804(8)$ & $3.850(25)$ \\
Bag model[25] & 3.511 & 3.630 & 3.664 & 3.764 \\
HQET[26] & 3.610 & 3.680 & 3.710 & 3.760 \\
Skyrmion model [27] & 3.752 & 3.793 & 3.934 & 3.953 \\
\hline
\end{tabular}

less massive than $\Omega_{c}^{*}$.

The results for singly charmed baryons are given in Table 44. Overall the results are in reasonable close to the experimental values where they are known.

The masses of doubly charmed baryons were also calculated. There are no experimental data but a comparison with a selection of model calculations is given in Table 5 (A more complete tabulation from various models may be found in |24|.) As might expected, without experimental constraints, the model calculations vary over a considerable range. The mass splittings from our quenched QCD simulation, 84(13)(5) MeV for $\Xi_{c c}^{*}-\Xi_{c c}$ and 78(7)(5)MeV for $\Omega_{c c}^{*}-\Omega_{c c}$, are substantial and lie in the middle of the range covered by the 
models listed in Table 5. They are only slightly smaller than the hyperfine splittings found for singly charmed baryons.

\section{Summary}

Hadron masses were calculated with an improved action on an anisotropic lattice with a spatial lattice spacing of about $0.2 \mathrm{fm}$. Comparison with simulations done at small lattice spacings and extrapolated to the continuum indicate that lattice spacing errors are less than $10 \%$.

The focus of this study is charmed baryons. For both singly and doubly charmed baryons spin splittings were found to be in agreement with expectations of quark models and other phenomenological approaches. The splittings for singly charmed baryons are somewhat larger than experimental values. This is in contrast to the small hyperfine effects for singly charmed baryons reported by the UKQCD collaboration [1].

The calculations reported here were done on a small lattice at a relatively large lattice spacing. By doing a unified study for light and heavy quark masses and comparing to continuum results where possible we have some confidence that the correct qualitative pattern of hyperfine effects in charmed baryons has been established for quenched lattice QCD. For a precise calculation, finite volume and lattice spacing issues have to be addressed. It is hoped that this can be done in the near future.

\section{Acknowledgements}

It is a pleasure to thank H.R. Fiebig, D.B. Leinweber, R. Lewis, N.H. Shakespeare and H.D. Trottier for help and discussion and B.K. Jennings for the use of his computers. This work is supported in part by the Natural Sciences and Engineering Research Council of Canada. 


\section{References}

[1] UKQCD Collaboration, K.C. Bowler et al., Phys. Rev. D 54 (1996) 3619 .

[2] A. Martin, J.-M. Richard, Phys. Lett. B 355 (1995) 345; B 185 (1983) 453.

[3] A. Ali Khan, T. Bhattacharya, S. Collins, C.T.H. Davies, R. Gupta, C. Morningstar, J. Shigemitsu, J. Sloan, Heavy-light Mesons and Baryons with b quarks, hep-lat/9912034.

[4] H.R. Fiebig, R.M. Woloshyn, Phys. Lett. B 385 (1996) 273; S. Collins, R.G. Edwards, U.M. Heller, J. Sloan, Nucl. Phys. B(Proc. Suppl.) 53 (1997) 877;F.X. Lee, D.B. Leinweber, Phys.Rev.D 59 (1999) 074504

[5] CP-PACS Collaboration, S. Aoki et al., Quenched Light Hadron Spectrum, hep-lat/9904012.

[6] UKQCD Collaboration, K.C. Bowler et al., Quenched QCD with $O(a)$ improvement: I. The spectrum of light hadrons, hep-lat/9910022.

[7] M. Benmerrouche, R.M. Davidson, N.C. Mukhopadhyay, Phys. Rev. C 39 (1989) 2339.

[8] E. Bagan, C. Chabab, H.G. Dosch, S. Narison, Phys. Lett. B 287 (1992) 176; B 278 (1992) 367.

[9] F. Karsch, Nucl. Phys. B 205[FS5] (1982) 285.

[10] M. Alford, T.R. Klassen, G.P. Lepage, Nucl.Phys. B 496 (1997) 377.

[11] D.B. Leinweber, R.M. Woloshyn, T. Draper, Phys. Rev. D 43 (1991) 1659.

[12] B.L. Ioffe, Nucl. Phys. B 188 (1981) 371 (E:B 191 (1981) 591).

[13] E.V. Shuryak, Nucl. Phys. B 198 (1982) 83.

[14] A.G. Grozin, O.I. Yakovlev, Phys. Lett. B 285 (1992) 254.

[15] C. Alexandrou, S. Güsken, F. Jegerlehner, K, Schilling, R. Sommer, Nucl. Phys. B 414 (1994) 815. 
[16] R.G. Edwards, U.M. Heller, T.R. Klassen, Nucl.Phys. B 517 (1998) 377.

[17] C. Morningstar, Nucl. Phys. B(Proc. Suppl.) 53 (1997) 914; N.H. Shakespeare, H.D. Trottier, Phys.Rev. D 59 (1999) 014502.

[18] C. Caso et al.(Particle Data Group), Eur. Phys. J. C 3 (1998) 1.

[19] UKQCD Collaboration, P. Boyle, The heavy quarkonium spectrum from quenched lattice QCD, hep-lat/9903017.

[20] H.D. Trottier, Phys.Rev. D 55 (1997) 6844; T.R. Klassen, Nucl. Phys. B(Proc. Suppl.) 73 (1999) 918.

[21] R. Lewis, R.M. Woloshyn, Phys. Rev. D 58 (1998) 074506.

[22] CLEO Collaboration, C.P. Jessop et al., Phys. Rev. Lett. 82 (1999) 492.

[23] S.S. Gershtein, V.V. Kiselev, A.K. Likhoded, A.I. Onishchenko, Mod. Phys. Lett. A 14 (1999) 135.

[24] L. Burakovsky, T. Goldman, L.P. Horwitz, Phys.Rev. D 56 (1997) 7124.

[25] W.A. Ponce Phys. Rev. D 19 (1982) 2197.

[26] J.G. Körner, M. Krämer, D. Pirjol, Prog. Part. Nucl. Phys. 33 (1994) 787.

[27] M. Rho, D.O. Riska, N.N. Scoccola, Phys. Lett. B 251 (1990) 597.

\section{Figure caption}

Fig. 1 Correlation function for the $\Omega_{c}$ field (squares) and the $\Omega_{c}^{*}$ field ( spin $3 / 2$ projection (triangles), spin $1 / 2$ projection (circles)) as a function of lattice time. 


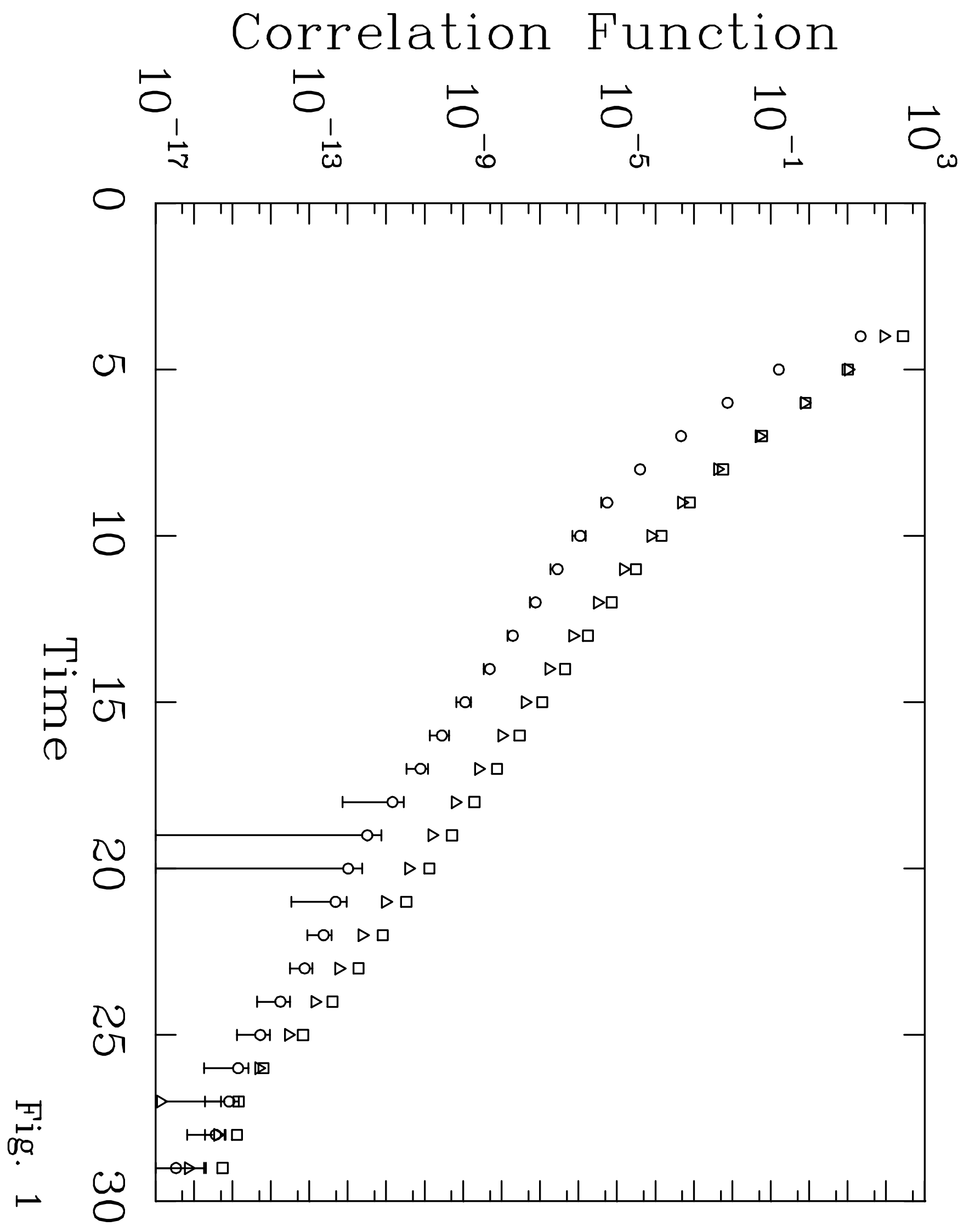

